\title{
Freezing in turbulent flow inside tubes and channels
}

\author{
B. Weigand and $\mathbf{H}$. Beer, Darmstadt, BRD
}

\begin{abstract}
A simple and quite flexible numerical model is presented to predict the steady state ice-layer formation inside a cooled two dimensional channel or a tube containing a turbulent flow. The effects of arbitrary entrance velocity distributions upon the shape of the ice-layers are examined. The presented numerical scheme is verified by comparing the predicted ice-layers with measurements and generally good agreement was found.
\end{abstract}

\section{Gefrieren in turbulent durchströmten Rohren und Kanälen}

Zusammenfassung. Es wurde ein einfaches und sehr flexibles numerisches Modell entwickelt, das es ermöglicht, die stationären Erstarrungsfronten im turbulent durchströmten ebenen Kanal und im Kreisrohr zu berechnen. Der Artikel befaßt sich unter anderem mit dem Einfluß des Eintrittsgeschwindigkeitsprofils auf die Kontur der erstarrten Schicht. Die Güte des vorgestellten Modells wurde anhand von eigenen Messungen für die Strömung mit Eisbildung im ebenen Kanal überprüft. Es zeigte sich eine sehr gute Übereinstimmung zwischen Theorie und Experiment.

\section{Nomenclature}

thermal diffusivity

dimensionless freezing parameter defined by Eq. (39) hydraulic diameter: $D=4 h$ (channel), $D=2 R$ (tube) modified stream functions

distance from centerline to the wall thermal conductivity

characteristic length: $L=h$ (channel), $L=R$ (tube) mixing length

pressure

Prandtl number turbulent Prandtl number

Reynolds number based on $L: R e_{L}=\bar{u}_{0} L / v$

Reynolds number based on the hydraulic diameter pipe radius

temperature

freezing temperature of the liquid

constant inlet temperature of the liquid

wall temperature

velocity components

mean axial velocity at the inlet

shear velocity

coordinates

dimensionless distance from the wall: $y^{+}=(\delta-n) / L u_{*} / v$ distance from centerline to the liquid-solid interface

dimensionless temperature

eddy viscosity

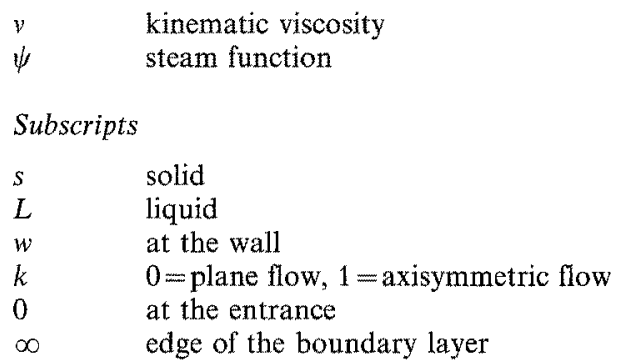

Superscripts

$\sim_{-}^{-} \quad$ dimensionless quantity

\section{Introduction}

Ice-formation phenomena for flowing water in a pipe or a channel, whose wall is kept at a uniform temperature below the freezing temperature of the water, is a basic engineering problem. It introduces many practical problems, such as pressure drop, diminution of flow rate and, sometimes, breakage of the pipe as a result of flow blockage by ice. If solidification on the cooled walls can not be suppressed, steady-state conditions must be aimed. Many theoretical and experimental studies have been performed for fluid flow with solidification in circular tubes and parallel plate channels and its effect upon laminar-flow heat transfer [1-4]. On the other hand, there are only few studies which deal with the prediction of ice-layers in the presence of turbulent flow. In many of these existing investigations it was assumed that the axial velocity profile will be similar to the fully developed turbulent entrance velocity profile throughout the whole axial region. By applying this assumption and with the use of an appropriate coordinate transformation, the energy equation can be reduced to a turbulent Graetz problem without solidification $[5,6]$. In the theoretical treatment of solidification in turbulent flow it was generally assumed, that the increase in ice-layer thickness in flow direction is small, so that the ice-layer is smooth and grows monotonously in thickness with increasing axial distance from the entrance. 
This assumption is correct, if the acceleration of the fluid in the entrance section, because of converging ice-layers, is not so pronounced that laminarization of the fluid occurs [10].

To our knowledge, there exists no numerical treatment of the conservation equations for turbulent flow with solidification inside a tube or a parallel plate channel.

Just as scarcely as theoretical studies on turbulent freezing inside a tube or channel, are experimental investigations which deal with smooth ice-layers in turbulent flow. There are many difficulties for direct measurement of the thickness of a smooth ice-layer inside a pipe. Therefore, there exist only measurements of the pressure drop and the heat flux at the pipe wall as a function of the axial distance $[7,8]$. In the case of ice-formation for water flow between two cooled plates, only one experimental study [9] is known.

The subject of this paper is the presentation of a simple and quite flexible numerical model for the prediction of steady-state ice layers inside a cooled pipe or a parallel plate channel containing a turbulent flow. The investigation deals solely with smooth ice-layers, which means that laminarization phenomena in the entrance region of the duct are excluded. The effect of the entrance velocity profile on the shape of the ice-layers is examined by performing the calculations both with a fully developed turbulent velocity profile at the entrance of the cooling section and with a slug flow profile.

\section{Analysis}

\subsection{Basic equations and assumptions}

Figure 1 shows the geometrical configuration and the coordinate system. The characteristic length $L$ denotes half of the channel height $h$ for the flow in a parallel plate channel and the radius $R$ for the flow in a circular tube. The turbulent fluid flow enters the cooled section at $x=0$ with an arbitrary velocity profile and with constant temperature $T_{0}$. In the chill region the wall temperature is maintained at a constant value $T_{W}$, which is lower than the freezing temperature $T_{F}$ of the fluid and a frozen layer is generated at the cooled wall. By assuming an incompressible, Newtonian fluid with constant fluid properties, the steady-state conservation equations for the fluid are cast into nondimensional form by use

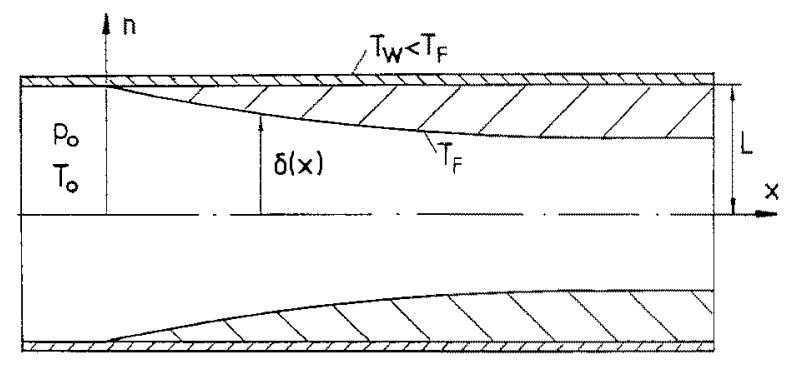

Fig. 1. Physical model and coordinate system of the following variables

$$
\begin{aligned}
& \tilde{x}=\frac{x}{L} ; \tilde{n}=\frac{n}{L} \sqrt{\operatorname{Re}_{L}} ; \tilde{\delta}=\frac{\delta}{L} ; \tilde{u}=\frac{u}{\bar{u}_{0}} ; \tilde{v}=\frac{v}{\bar{u}_{0}} \sqrt{\operatorname{Re}_{L}} ; \tilde{r}=\frac{r}{L} \\
& \tilde{p}=\frac{p}{\varrho \bar{u}_{0}^{2}} ; \varepsilon_{m}^{+}=\frac{\varepsilon_{m}}{v} ; \operatorname{Re}_{L}=\frac{\bar{u}_{0} L}{v} ; \operatorname{Pr}=\frac{v}{a} ; \operatorname{Pr}_{t}=\frac{\varepsilon_{m}}{\varepsilon_{h}} ; \theta=\frac{T-T_{F}}{T_{0}-T_{F}}
\end{aligned}
$$

This results in

$\frac{\partial}{\partial \tilde{x}}\left(\tilde{r}^{k} \tilde{u}\right)+\frac{\partial}{\partial \tilde{n}}\left(\tilde{r}^{k} \tilde{v}\right)=0$

$\tilde{u} \frac{\partial \tilde{u}}{\partial \tilde{x}}+\tilde{v} \frac{\partial \tilde{u}}{\partial \tilde{n}}=-\frac{\partial \tilde{p}}{\partial \tilde{x}}+\frac{1}{\tilde{r}^{k}} \frac{\partial}{\partial \tilde{n}}\left[\tilde{r}^{k}\left(1+\varepsilon_{m}^{+}\right) \frac{\partial \tilde{u}}{\partial \tilde{n}}\right]$

$\frac{\partial \tilde{p}}{\partial \tilde{n}}=0$

$\tilde{u} \frac{\partial \theta}{\partial \tilde{x}}+\tilde{v} \frac{\partial \theta}{\partial \tilde{n}}=\frac{1}{P r} \frac{1}{\tilde{r}^{k}} \frac{\partial}{\partial \tilde{n}}\left[\tilde{r}^{k}\left(1+\frac{P r}{P r_{t}} \varepsilon_{m}^{+}\right) \frac{\partial \theta}{\partial \tilde{n}}\right]$

By deriving the Eqs. (2)-(5), the usual boundary-layer assumptions were made [11], [12]. The flow index $k$, which appears in Eqs. (2)-(5), is zero for plane flow and unity for axisymmetric flow. The boundary conditions belonging to the conservation equations are:

$\tilde{x}=0: \quad \tilde{p}=\tilde{p}_{0} ; \quad \tilde{u}=$ given $; \quad \tilde{v}=$ given $; \quad \theta=1$

$\tilde{n}=0: \quad \frac{\partial \tilde{u}}{\partial \tilde{n}}=0 ; \quad \tilde{v}=0 ; \quad \frac{\partial \theta}{\partial \tilde{n}}=0$

$\tilde{n}=\sqrt{\operatorname{Re}_{L}} \tilde{\delta} ; \quad \tilde{u}=\tilde{v}=0 ; \quad \theta=0$

In addition to Eqs. (2)-(6) the energy equation for the solid region is required. By assuming constant properties in the solid region and negligible axial conduction, the heat conduction equation for the solid-phase reduces to

$\frac{\partial}{\partial n}\left(r^{k} \frac{\partial T_{s}}{\partial n}\right)=0$

with the following boundary conditions:

$T_{s}(n=\delta)=T_{F}$

$T_{s}(n=L)=T_{W}$

Equations (2)-(6) and Equations (7)-(8) are coupled by the interface energy equation which takes the following form for steady-state conditions

$k_{s} \frac{\partial T_{s}}{\partial n}=k_{L} \frac{\partial T}{\partial n} ; \quad n=\delta$

\subsection{Temperature distribution in the solid region}

With the boundary condition Eq. (8), the temperature distribution in the solid phase is easily calculated from Eq. (7). For 
$k=0$ (channel) this results in

$T_{s}=T_{W} \frac{n-\delta}{h-\delta}+T_{F} \frac{h-n}{h-\delta}$

and for $k=1$ (tube)

$T_{S}=T_{W}+\left(T_{F}-T_{W}\right) \frac{\ln (\tilde{r})}{\ln (\tilde{\delta})}$

\subsection{Velocity and temperature distribution in the liquid}

To ensure that Eqs. (2)-(5) have a form that is common to plane and axisymmetric flows, the Mangler transformation

$d \tilde{x}=d \tilde{x} ; \quad d \bar{n}=(\tilde{r})^{k} d \tilde{n}$

is applied to Eqs. (2)-(5). This results in the ensueing set of equations:

$\frac{\partial \bar{u}}{\partial \bar{x}}+\frac{\partial \bar{v}}{\partial \bar{n}}=0$

$\bar{u} \frac{\partial \bar{u}}{\partial \bar{x}}+\bar{v} \frac{\partial \bar{u}}{\partial \bar{n}}=-\frac{\partial \tilde{p}}{\partial \bar{x}}+\frac{\partial}{\partial \bar{n}}\left[b_{1} \frac{\partial \bar{u}}{\partial \bar{n}}\right]$

$\frac{\partial \tilde{p}}{\partial \tilde{n}}=0$

$\ddot{u} \frac{\partial \theta}{\partial \bar{x}}+\bar{v} \frac{\partial \theta}{\partial \bar{n}}=\frac{1}{\operatorname{Pr}} \frac{\partial}{\partial \bar{n}}\left[b_{2} \frac{\partial \theta}{\partial \bar{n}}\right]$

with the abbreviations

$b_{1}=\tilde{r}^{2 k}\left(1+\varepsilon_{m}^{+}\right) ; \quad b_{2}=\tilde{r}^{2 k}\left(1+\frac{\operatorname{Pr}}{\operatorname{Pr}_{t}} \varepsilon_{m}^{+}\right)$

After introducing a stream function, defined as

$\bar{u}=-\frac{\partial \psi}{\partial n} ; \quad \bar{v}=\frac{\partial \psi}{\partial \bar{x}}$

into the Eqs. (13)-(16) and by applying the following coordinate transformation

$\tilde{y}=\frac{\sqrt{\operatorname{Re}_{L}}}{k+1}\left[1-\left(\frac{n}{\hat{\delta}}\right)^{k+1}\right] ; \quad \bar{x}=\tilde{x}$

to the conservation equations, the following set of partial differential equations can be obtained

$\frac{1}{\bar{\delta}^{2}} \frac{\partial}{\partial \tilde{y}}\left[b_{1} \frac{\partial^{2} F}{\partial \tilde{y}^{2}}\right]$

$=\frac{\partial \tilde{p}}{\partial \bar{x}}+\frac{\partial F}{\partial \tilde{y}} \frac{\partial^{2} F}{\partial \bar{x} \partial \tilde{y}}-\frac{\partial F}{\partial \bar{x}} \frac{\partial^{2} F}{\partial \tilde{y}^{2}}-\frac{(k+1)}{\tilde{\delta}} \frac{d \tilde{\delta}}{d \bar{x}} F \frac{\partial^{2} F}{\partial \tilde{y}^{2}}$

$\frac{\partial \tilde{p}}{\partial \tilde{y}}=0$

$\frac{1}{\widetilde{\delta}^{2}} \frac{\partial}{\partial \tilde{y}}\left[\bar{b}_{2} \frac{\partial \theta}{\partial \tilde{y}}\right]=\operatorname{Pr}\left[\frac{\partial F}{\partial \tilde{y}} \frac{\partial \theta}{\partial \bar{x}}-\frac{\partial F}{\partial \bar{x}} \frac{\partial \theta}{\partial \tilde{y}}-\frac{(k+1)}{\tilde{\delta}} \frac{d \tilde{\delta}}{d \bar{x}} F \frac{\partial \theta}{\partial \tilde{y}}\right]$ with the modified stream function

$F=\frac{\psi}{\tilde{\delta}^{k+1}}$

and the abbreviations

$\bar{b}_{1}=\left(\frac{r}{\delta}\right)^{2 k}\left(1+\varepsilon_{m}^{+}\right)$

$\bar{b}_{2}=\left(\frac{r}{\delta}\right)^{2 k}\left(1+\frac{P r}{\operatorname{Pr}_{t}} \varepsilon_{m}^{+}\right)$

The coordinate transformation according to Eq. (19) is very useful in order to solve the conservation equations, because the duct with variable distance between the wall and the centerline $\tilde{\delta}(\bar{x})$ is transformed into a duct with constant height.

The boundary conditions for Eqs، (20)-(22) are given by

$\bar{x}=0: \quad F=$ given $; \quad \tilde{p}=\tilde{p}_{0} ; \quad \theta=1$

$\tilde{y}=0: \quad \frac{\partial F}{\partial \tilde{y}}=0 ; \quad F=0 ; \quad \theta=0$

$\tilde{y}=\tilde{y}_{c}: \quad \frac{\partial^{2} F}{\partial \tilde{y}^{2}}=0 ; \quad \frac{\partial \theta}{\partial \tilde{y}}=0 \quad$ (channel)

$\tilde{y}=\tilde{y}_{c}: \quad \frac{-2}{\sqrt{R e_{L}}} \frac{\partial^{2} F}{\partial \tilde{y}^{2}}=\widetilde{\delta}^{2}\left(\frac{\partial \tilde{p}}{\partial \tilde{x}}+\frac{1}{2} \frac{\partial}{\partial \bar{x}}\left[\frac{\partial F}{\partial \tilde{y}}\right]^{2}\right)$

$\frac{-2}{\sqrt{R e_{L}}} \frac{\partial \theta}{\partial \tilde{y}}=\operatorname{Pr} \widetilde{\delta}^{2} \frac{\partial F}{\partial \tilde{y}} \frac{\partial \theta}{\partial \bar{x}} \quad$ (tube)

where $\tilde{y}_{c}=\sqrt{R e_{L}} /(k+1)$ denotes the distance between the centerline of the duct and the liquid-solid interface.

By deriving the boundary conditions at the centerline for the circular tube in transformed coordinates, the evaluation of the terms $\partial \tilde{u} / \partial \tilde{n}=0$ and $\partial \theta / \partial \tilde{n}=0$ results in indeterminate expressions of the form $0 / 0$. This is due to the use of Mangler transformed coordinates. Therefore, the boundary conditions at the centerline have to be calculated by utilizing Eqs. (20)-(22). For $\widetilde{\delta}=1$, the given boundary conditions reduce to the expressions for a pipe without solidification [11].

Equations (20)-(22) were derived in physical coordinates which are appropriate in the case of a fully developed velocity profile at the entrance of the chill region. For the case of combined hydrodynamic and thermal development of the flow, which is given by a slug flow profile at the entrance of the cooling section, however, it is convenient to take Falkner-Skan transformed variables [12].

$\eta=\frac{\tilde{y}}{\sqrt{\bar{x}}} ; \quad \bar{x}=\tilde{x}$

Applying the coordinate transform to the conservation equations and inserting a modified stream-function

$F(\bar{x}, \tilde{y})=\sqrt{\bar{x}} f(\bar{x}, \eta)$ 
results in the following equations

$\frac{1}{\widetilde{\delta}^{2}} \frac{\partial}{\partial \eta}\left[\bar{b}_{1} \frac{\partial^{2} f}{\partial \eta^{2}}\right]+\frac{1}{2} f \frac{\partial^{2} f}{\partial \eta^{2}}$

$=\bar{x} \frac{\partial \tilde{p}}{\partial \bar{x}}+\bar{x}\left[\frac{\partial f}{\partial \eta} \frac{\partial^{2} f}{\partial \bar{x} \partial \eta}-\frac{\partial f}{\partial \bar{x}} \frac{\partial^{2} f}{\partial \eta^{2}}-\frac{(k+1)}{\widetilde{\delta}} \frac{d \tilde{\delta}}{d \bar{x}} f \frac{\partial^{2} f}{\partial \eta^{2}}\right]$

$\frac{\partial \tilde{p}}{\partial \eta}=0$

$\frac{1}{\widetilde{\delta}^{2}} \frac{\partial}{\partial \eta}\left[\bar{b}_{2} \frac{\partial \theta}{\partial \eta}\right]+\frac{\operatorname{Pr}}{2} f \frac{\partial \theta}{\partial \eta}$

$=\bar{x} \operatorname{Pr}\left[\frac{\partial f}{\partial \eta} \frac{\partial \theta}{\partial \bar{x}}-\frac{\partial f}{\partial \bar{x}} \frac{\partial \theta}{\partial \eta}-\frac{(k+1)}{\tilde{\delta}} \frac{d \tilde{\delta}}{d \bar{x}} f \frac{\partial \theta}{\partial \eta}\right]$

The boundary conditions belonging to Eqs. (28)-(30) have to be formulated at the liquid-solid interface $(\eta=0)$ and at the edge of the boundary layer $\left(\eta=\eta_{\infty}\right)$

$\eta=0: \quad f=\frac{\partial f}{\partial \eta}=0 ; \quad \theta=0$

$\eta=\eta_{\infty}: \quad \frac{\partial^{2} f}{\partial \eta^{2}}=0 ; \quad \frac{\partial \theta}{\partial \eta}=0$

The initial conditions for $\bar{x}=0$ remains unchanged.

In addition to the boundary conditions, the conservation of mass in integral form must be satisfied

$\bar{u}_{0} L^{k+1}=\frac{1}{k+1} \int_{0}^{\delta} u r^{k} d n$

Equation (32) results in an expression for the modified stream-function at the centerline of the duct in physical coordinates

$F\left(\bar{x}, \tilde{y}_{c}\right)=\frac{\sqrt{R e_{L}}}{(k+1) \tilde{\delta}^{k+1}}$

or at the boundary layer edge in transformed coordinates

$\sqrt{\frac{R e_{L}}{\bar{x}}} \frac{1}{(k+1)} \frac{1}{\tilde{\delta}^{k+1}}=f\left(\bar{x}, \eta_{\infty}\right)+\left.\frac{\partial f}{\partial \eta}\right|_{\eta_{\infty}}\left(\sqrt{\frac{R e_{L}}{\bar{x}}} \frac{1}{(k+1)}-\eta_{\infty}\right)$

The energy balance at the liquid-solid interface, Eq. (11), can be rewritten after inserting the temperature distribution in the solid phase in physical coordinates

$\left.\frac{\sqrt{\operatorname{Re}_{L}}}{B} \frac{\partial \theta}{\partial \tilde{y}}\right|_{\tilde{y}=0}=\frac{\tilde{\delta}}{1-\tilde{\delta}} \quad$ (channel)

$\left.\frac{\sqrt{R e_{L}}}{B} \frac{\partial \theta}{\partial \tilde{y}}\right|_{\hat{\mathbf{y}}=0}=\frac{1}{\ln \tilde{\delta}} \quad$ (tube)

and in transformed variables

$\left.\sqrt{\frac{R e_{L}}{\bar{x}}} \frac{1}{B} \frac{\partial \theta}{\partial \eta}\right|_{\eta=0}=\frac{\tilde{\delta}}{1-\tilde{\delta}} \quad$ (channel)
$\left.\sqrt{\frac{R e_{L}}{\bar{x}}} \frac{1}{B} \frac{\partial \theta}{\partial \eta}\right|_{\eta=0}=\frac{1}{\ln \tilde{\delta}} \quad$ (tube)

with the dimensionless freezing parameter $B$, defined as

$B=\frac{k_{s}}{k_{L}} \frac{T_{F}-T_{W}}{T_{0}-T_{F}}$

\subsubsection{Physical assumptions for turbulent flow}

For turbulent flows in ducts, there are essentially three distinct regions. If a slug flow velocity profile is introduced at the entrance of the chill region, the shear layers develop in the region close to the solid wall. The core velocity increases continuously because of the growth in displacement thickness and as a result of the converging ice-layers at the walls of the duct. This region can be described by the two-layer model developed by Cebeci and Smith [13]. The eddy-viscosity in the near wall region is given by

$\left(\varepsilon_{m}\right)_{i}=l^{2}\left|\frac{\partial u}{\partial n}\right| ; \quad\left(\varepsilon_{m}\right)_{i}<\left(\varepsilon_{m}\right)_{0}$

$l=\varkappa \delta\left(1-\frac{n}{\delta}\right)\left[1-\exp \left(-\frac{y^{+}}{26}\right)\right] ; \quad x=0.4$

In the outer layer the eddy-viscosity is assumed to be constant

$\left(\varepsilon_{m}\right)_{0}=\alpha\left|\int_{0}^{\infty}\left(u_{\infty}-u\right) r^{k} d n\right| ; \quad \alpha=0.0168$

As the flow moves downstream, the shear layers grow and finally meet at the centerline. In this region (region III), the well-known mixing-length formula of Nikuradse with the damping factor according to van Driest is used to model $\varepsilon_{m}$

$\varepsilon_{m}=l^{2}\left|\frac{\partial u}{\partial n}\right|$

with $l$ given by

$l=\delta\left[0.14-0.08\left(\frac{n}{\delta}\right)^{2}-0.06\left(\frac{n}{\delta}\right)^{4}\right]\left(1-\mathrm{e}^{-y^{+} / 26}\right)$

By deriving the mixing length expression according to Eqs. (41) and (44) it is assumed that the mixing length distribution behaves similar to that in a straight duct by scaling it with the local duct height $\delta(x)$.

For the region between the displacement-interaction and fully developed region, the eddy viscosity formula can be modelled by the following expression according to [12].

$\left(\varepsilon_{m}\right)_{\mathrm{II}}=\left(\varepsilon_{m}\right)_{\mathrm{I}}+\left[\left(\varepsilon_{m}\right)_{\mathrm{III}}-\left(\varepsilon_{m}\right)_{\mathrm{I}}\right]\left(1-\exp \left(-\frac{\bar{x}-\bar{x}_{0}}{20}\right)\right)$

with $\left(\varepsilon_{m}\right)_{\mathrm{I}}$ denoting the formula given by Eqs. (40)-(42) and $\left(\varepsilon_{m}\right)_{\mathrm{III}}$ the formula given by Eqs. (43), (44). $\bar{x}_{0}$ defines the axial position for which the shear layers have reached $95 \%$ of the distance between centerline and liquid-solid interface. Cebeci and Chang [12] performed many calculations with 
the help of the above given turbulence model, concerning the hydrodynamic and thermal development of flows in parallel plate channels and circular tubes without solidification at the walls. They checked their numerical solutions by comparing with experimental results. Generally good agreement was found. In order to relate the eddy diffusivity of heat, which appears in the energy equation, to the eddy viscosity $\varepsilon_{m}$, a formulation of the turbulent Prandtl number is needed. In the following calculations the turbulent Prandtl number concept from Cebeci and Smith [13] was used.

\section{The numerical method}

The calculation of the frozen layer can be performed in the following manner: an arbitrary distribution for $\bar{\delta}(\vec{x})$ is assumed, for example a linear decreasing function of $\bar{x}$. With the assumed distribution of $\widetilde{\delta}(\bar{x})$ Eqs. (20)-(22) or Eqs. (28)(30) can be solved numerically, according to their boundary conditions. After solving the conservation equations, a new distribution of $\tilde{\delta}(\bar{x})$ is calculated by inserting the yet known temperature gradient at the solid-liquid interface into one of the Eqs. (35)-(38). The iteration procedure described above was repeated until the deviation between two successive ice-layers was within $\Delta \widetilde{\delta}=\left|\widetilde{\delta}^{(i)}-\widetilde{\delta}^{(i+1)}\right|<0.01$ at every axial position.

Solutions of the conservation equations were obtained with the help of an implicite finite-difference method, which is known in literature as the Keller-box method [11]. This method has several very desirable features that make it appropriate for the solution of all parabolic partial differential equations. One of which is that it allows highly nonuniform $\bar{x}$ and $\bar{y}$ spacings by second order accuracy. Because the box scheme is a common method for solving parabolic differential equations, only a brief outline is provided here [11]. First of all the parabolic differential equation of order $n$ is reduced to a system of $n$ first-order equations. These equations were approximated by difference equations using central differences at each nodal point. The ensueing algebraic equations were linearized. This results in a linear system of equations, which show a block tridiagonal structure and can be solved easily.

The pressure gradient $\partial \tilde{p} / \partial \bar{x}$, which appears in Eq. (20) or Eq. (28), can be treated in two different ways [12]:

\section{- Nonlinear eigenvalue method}

By omitting Eq. (21) or (29), the pressure gradient, which appears in the momentum equation, introduces an additional unknown to the system given by Eqs. (2)-(4) and can be treated as a nonlinear eigenvalue [12]. Therefore, a pressure difference between two axial net points is assumed. With the help of this given pressure difference, the momentum equation can be solved. By checking the conservation of mass, according to Eq. (32), it can be distinguished whether the assumed pressure difference was correct. If Eq. (32) is violat- ed, a new value for the pressure difference is obtained by solving variational equations. This iteration is continued until the deviation is $\left|\Delta p^{(i+1)}-\Delta p^{(i)}\right|<10^{-4}$. For further details see $[11,12]$.

\section{- Mechul method}

In contrast to the nonlinear eigenvalue approach, the Mechul method solves directly for $\tilde{p}$ using Eq. (20) or Eq. (29). This makes iteration superfluous and results in four partial differential equations of the first order. Therefore, the algebra for solving this equations is a little more involved than in the case of the nonlinear eigenvalue approach.

The authors have applied both above mentioned solution procedures and found out, that they are nearly equivalent. However, the nonlinear eigenvalue method was found to require approx. $10 \%$ more CPU time than the Mechul method. Finally it should be stated that the iteration process converges rapidly in both cases. Normally only three iterations were necessary to get $\delta(\bar{x})$ within the above prescribed error tolerance. The calculations were performed with the help of a strongly nonuniform grid in $\bar{y}$-and $\bar{x}$-direction. Numerical runs showed that approx. 50 grid points in $\tilde{y}$-direction and $50-100$ points in axial direction guaranteed sufficient accuracy. The number of grid points in axial direction depends strongly on the length of the chill region.

\section{Results and discussion}

The described calculation procedure is able to predict a realistic variation of $\tilde{\delta}(\bar{x})$ with $\bar{x}$ as long as the interaction between the turbulent flow, the shape of the ice-layer and the heat transfer at the ice-water interface does not result in an instability of the ice-layer. Weigand and Beer [14] presented a detailed and comprehensive experimental investigation about the morphology of the ice structure in a parallel plate channel. They found out that the ice-layers remained smooth as long as

$\theta_{c}<-0.41+\frac{R e_{D}}{7077} \quad\left(R e_{D}>6000\right)$

Herein, $\theta_{c}$ is a dimensionless temperature ratio, defined by

$\theta_{c}=\frac{T_{W}-T_{F}}{T_{F}-T_{0}}$

For values of $\theta_{c}$ violating Eq. (46), the ice-layer gets instable and a wavy structure develops. In the following results it is generally assumed that the ice-layer is smooth, which means in the case of a parallel plate channel that the inequality of Eq. (46) holds.

\subsection{Fully developed entrance velocity profile}

Figure 2 shows the influence of increasing wall cooling on the ice-layer at the wall for a parallel plate channel. It is obvious that the ice-layer thickness increases with growing 


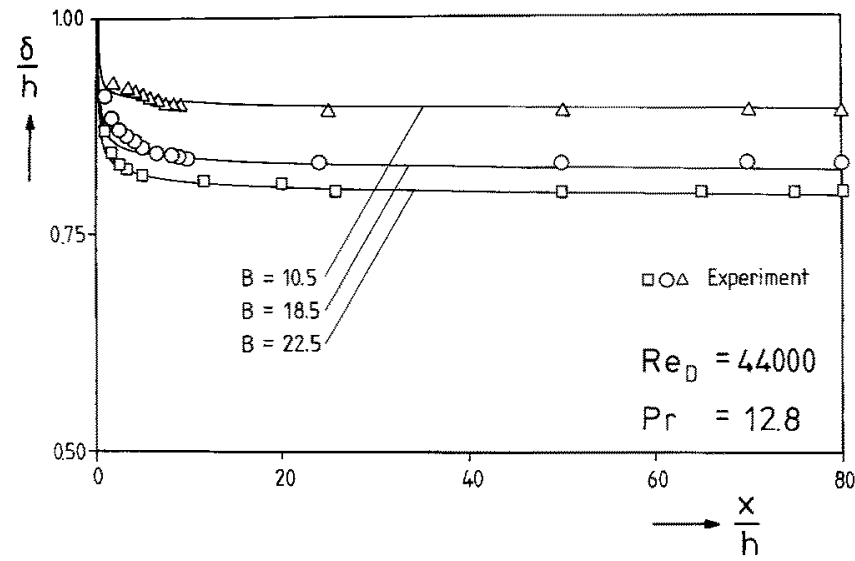

Fig. 2. Ice-layer thickness in a parallel plate channel for $R e_{D}=$ $44,000, P r=12.8$ and various $B$

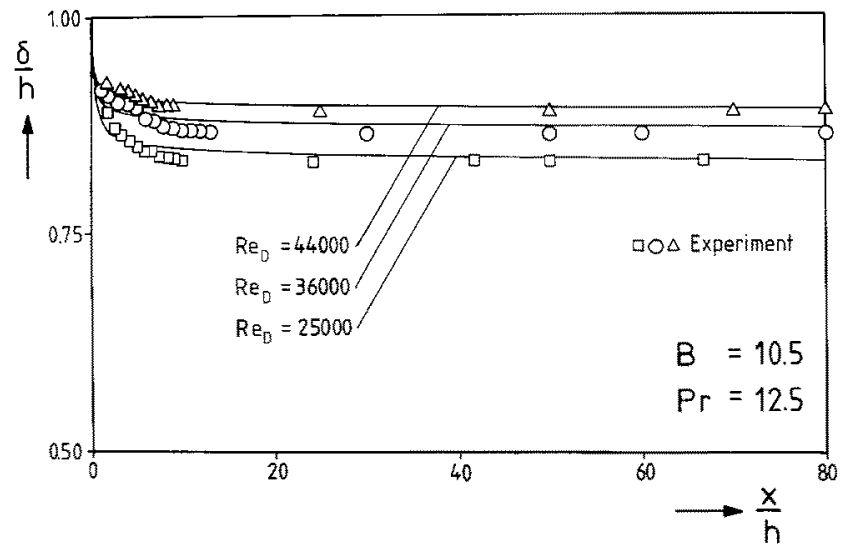

Fig. 3. Effect of Reynolds number on the axial distribution of $\tilde{\delta}$ in a parallel plate channel for $B=10.5$ and $\operatorname{Pr}=12.5$

$B$. This fact can be easily understood by examining the interface energy equation. Solving Eq. (35) for $\widetilde{\delta}$, one obtains

$\tilde{\delta}=1 /\left(1+\frac{B}{\sqrt{\operatorname{Re}_{L}}} \frac{1}{\partial \theta /\left.\partial \tilde{y}\right|_{\tilde{y}=0}}\right)$

By assuming a constant Reynolds number, the heat flux from the fluid to the ice-layer is nearly constant at a given axial position. Therefore, increasing values of $B$ result in a smaller distance between the centerline and the liquid-solid interface. Figure 3 elucidates the influence of the Reynolds number on the axial distribution of $\widetilde{\delta}$ for $B=10.5$ and $P r=12.5$ in a parallel plate channel. It can be seen, that an increasing Reynolds number results in a growing heat flux from the fluid to the solid-liquid interface and, therefore, in a decreasing ice-layer thickness. In Figs. 2 and 3 measurements of the ice-layer thickness in a parallel plate channel are shown for comparison. The measurements were performed by the authors in a parallel plate channel with $1800 \mathrm{~mm}$ in length and a half channel-height $h=12 \mathrm{~mm}$. The ice-layer thickness was measured with the help of a microscope, fastened sliding in front of the channel with three selectable enlargements.

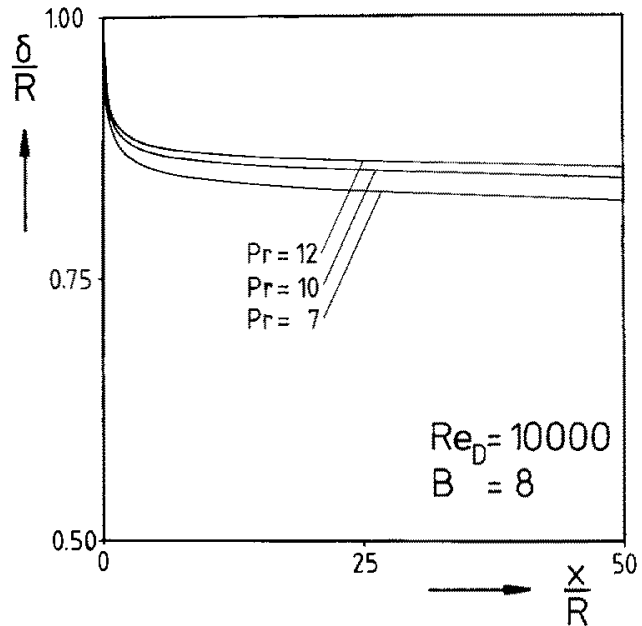

Fig. 4. Influence of Prandtl number on the shape of the ice-layer in a pipe for $R e_{D}=10,000$ and $B=8$

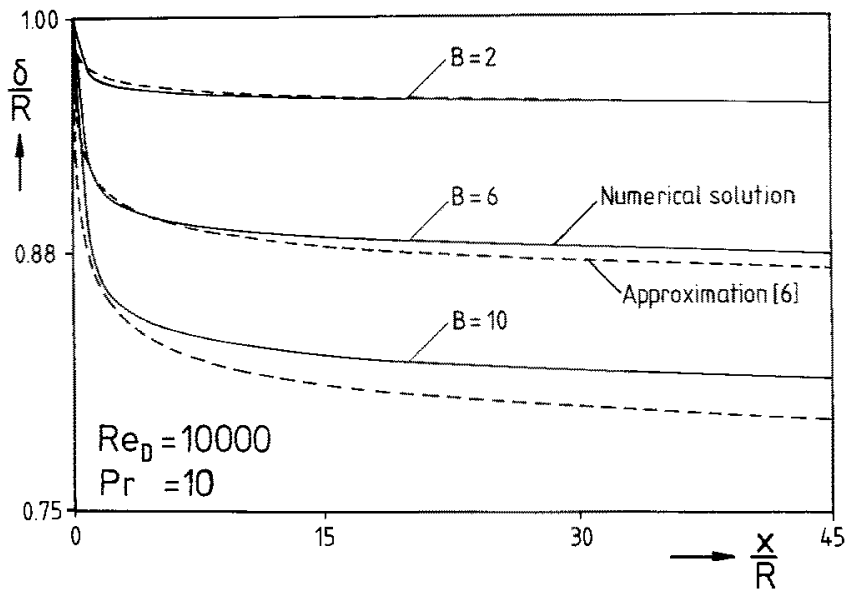

Fig. 5. Ice-layer thickness in a pipe for $R e_{D}=10,000, P r=10$ compared with the approximation given by Shibani and Özisik [6]

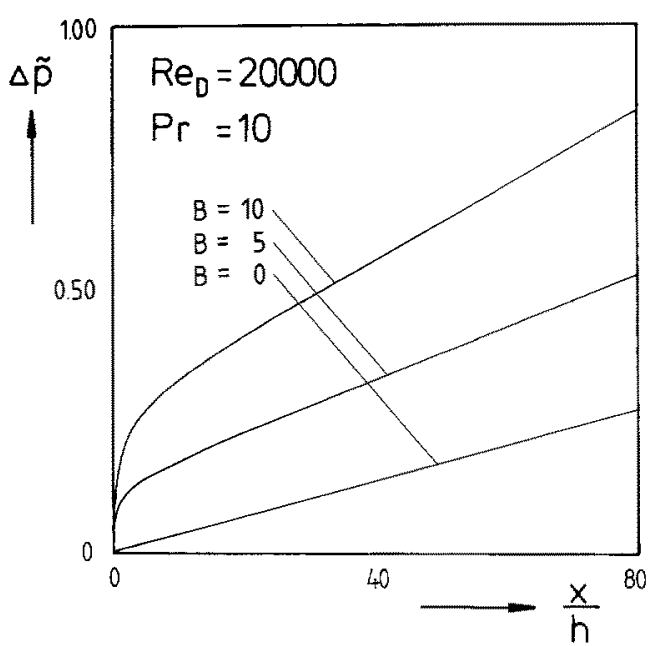

Fig. 6. Effect of freezing in a planar channel upon the pressure drop for $R e_{D}=20,000$ and $P r=10$ 


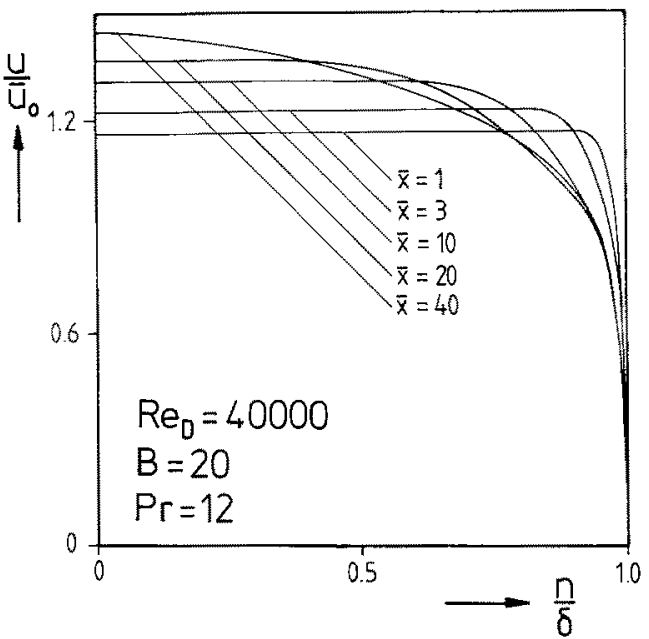

Fig. 7. Development of $u / \bar{u}_{0}$ for $R e_{D}=40,000, B=20$ and $P r=12$ in the combined hydrodynamic and thermal entrance region of a parallel plate channel

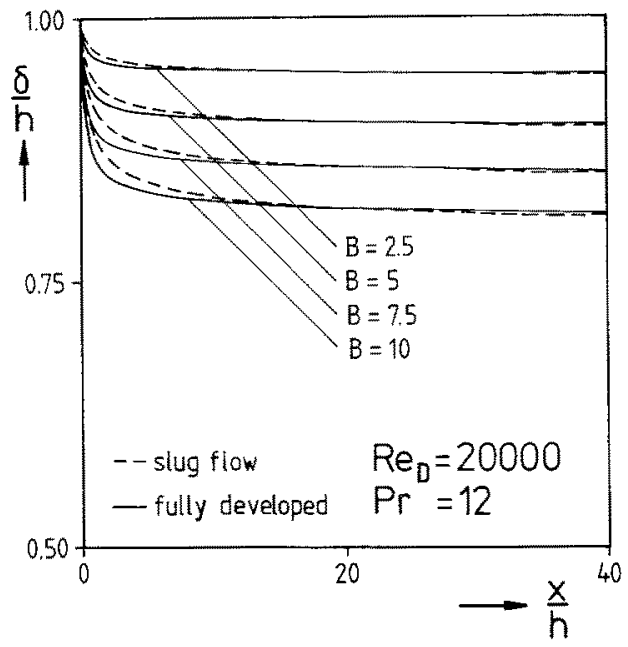

Fig. 8. Influence of the entrance velocity profile on the shape of the ice-layers in a two-dimensional channel

The ice-layer thickness could be measured with good accuracy of the order less than $0.1 \mathrm{~mm}$. For further details concerning the experimental apparatus and the operating procedure see [14]. As can be seen from Figs. 2, 3 the agreement between theory and experiment is very good. In Fig. 4, the effect of increasing Prandtl number on the shape of the ice-layer in a pipe is shown for $\operatorname{Re}_{D}=10,000$ and $B=8$. The ice-layer thickness decreases with increasing Prandtl number. This is due to the decreasing thermal boundary layer thickness, causing a steeper temperature gradient at the solid-liquid interface with growing Prandtl number. Figure 5 shows a comparison between numerical calculated ice-layers in a pipe and freezing fronts, which were determined by the approximation according to Shibani and Özisik [6]. It can be seen that the numerical solution leads to slightly thinner ice-layers than the approximate solution.

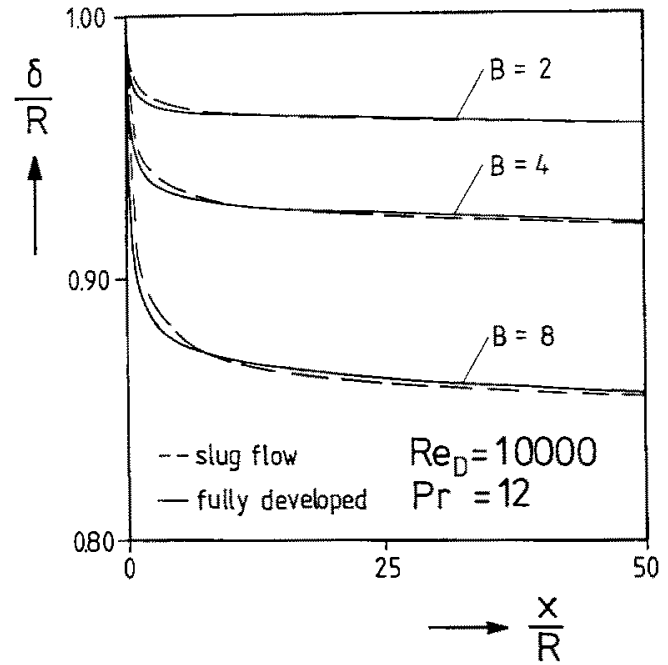

Fig. 9. Influence of the entrance velocity profile on the shape of the ice-layers in a pipe

However, the absolute deviation $\Delta \tilde{\delta}$ between the compared ice-layers is less than 0.02 . The effect of ice formation in a planar channel on the pressure drop is visualized in Fig. 6. It is obvious that the pressure drop increases sharply in the entrance region of the channel and approaches a linear growth for greater values of the axial coordinate. This can be understood if one recognizes that the ice-layers converge rapidly for low values of the axial coordinate and form a nearly parallel plate channel for greater values of $\bar{x}$.

\subsection{Combined hydrodynamic- and thermal development}

For the case of solidification in the presence of turbulent flow in the combined hydrodynamic- and thermal entrance region of a parallel plate channel or a pipe exists neither a theoretical nor an experimental investigation. Figure 7 shows the development of the axial velocity profile in a parallel plate channel for $R e_{D}=40,000$ and $B=20$. The acceleration of the fluid, caused by the converting ice-layers, gives rise to a more rapid development of the entering slug flow profile than in a channel without solidification. Figure 8 elucidates the influence of different entrance velocity profiles on the shape of the ice-layer in a parallel plate channel for $R e_{D}=$ 20,000 and $P r=12$. It can be seen that the ice-layers for combined hydrodynamic- and thermal development of the flow are slightly thinner than for a fully developed entrance velocity profile. This can be attributed to the enhancement of heat transfer between the liquid and the solid region due to steeper velocity gradients at the wall for a slug flow velocity profile at the entrance of the chill region. However, it is obvious that the difference in thickness between the two ice-layers for a fixed value of $B$ is very small and can be neglected with good accuracy. In Fig. 9, the effect of different entrance velocity profiles on the shape of the freezing front in a pipe is shown for $\operatorname{Re}_{D}=10,000$ and $\operatorname{Pr}=12$. It can be 
seen that also for pipe flow the influence of the entrance velocity profile on the shape of the ice-layer can be ignored.

\section{Concluding remarks}

A simple numerical model was presented to predict the steady-state ice-layers inside a parallel plate channel or a circular pipe containing a turbulent flow. The effect of arbitrary entrance velocity distribution upon the shape of the ice-layers was examined. It was shown that the axial distribution of $\tilde{\delta}$ is only slightly influenced by the entrance velocity profile. For the case of a fully developed entrance velocity profile in a two dimensional channel the numerical results were compared with experimental findings. A generally good agreement was found. Finally, it should be stated that the given model can be used to predict the velocity profiles and the heat transfer in an arbitrary shaped two dimensional channel or in a pipe.

\section{Acknowledgements}

The financial support of this work by the Deutsche Forschungsgemeinschaft is greatly acknowledged.

\section{References}

1. Zerkle, R. D.; Sunderland, J E.: The effect of liquid solidification in a tube upon laminar-flow heat transfer and pressure drop. J. Heat Transfer 90 (1968) 183-190

2. Lee, D. G.; Zerkle, R. D.: The effect of liquid solidification in a parallel plate channel upon laminar-flow heat transfer and pressure drop. J. Heat Transfer 91 (1969) 583-585

3. Chida, K.: Heat transfer in steady laminar pipe flow with liquid solidification. Heat Transfer: Jap. Res. 81 (1983) 81-94
4. Weigand, B.; Beer, H.: Heat transfer and solidification of a laminar liquid flow in a cooled parallel plate channel: The stationary case. Wärme-Stoffübertrag. 26 (1991) 233-240

5. Shibani, A. A.; Ozisik, M. N.: A solution of freezing of liquids of low Prandtl number in turbulent flow between parallel plates. Journal of Heat Transfer 99 (1977) 20-24

6. Shibani, A. A.; Özisik, M. N.: Freezing of liquids in turbulent flow inside tubes. Can. J. Chem. Eng. 55 (1977) 672-677

7. Thomason, S. B.; Mulligan, J. C.; Everhart, J.: The effect of internal solidification on turbulent flow heat transfer and pressure drop in a horizontal tube. J. Heat Transfer 100 (1978) $387-394$

8. Thomason, S. B.; Mulligan, J. C.; Hill: A simple quasi-steady analysis of turbulent flow freezing transients and comparison with experiments. Can. J. Chem. Eng. 67 (1989) 368-377

9. Seki, N.; Fukusako, S.; Younan, G. W.: Ice-formation phenomena for water flow between two cooled parallel plates. J. Heat Transfer 106 (1984) 498-505

10. Gilpin, R. R.: Ice formation in a pipe containing flows in the transition and turbulent regimes. J. Heat Transfer 103 (1981) $363-368$

11. Cebeci, T.; Bradshaw, P.: Physical and computational aspects of convective heat transfer. Berlin, Heidelberg, New York: Springer 1984

12. Cebeci, T.; Chang, K. C.: A general method for calculating momentum and heat transfer in laminar and turbulent flows. Num. Heat Transfer 1 (1978) 39-68

13. Cebeci, T.; Smith, A. M. O.: Analysis of turbulent boundary layers. New York: Academic Press 1974

14. Weigand, B; Beer, H.: The morphology of ice structure in a parallel plate channel. Proceedings Third International Symposium on Cold Regions Heat Transfer, Jun 12-14, 1991, Fairbanks, Alasca, USA

Prof. Dr.-Ing. H. Beer

Dipl.-Ing. B. Weigand

Institut für Technische Thermodynamik

Petersenstraße 30

W-6100 Darmstadt

Received April 26, 1991 